\title{
Lesión en la hipofaringe por un desplazamiento del arco de brackets. Reporte de caso
}

\author{
Hypopharyngeal injury due to displacement of brackets arch. Case report
}

\author{
Dra. María S. Labadens ${ }^{a}$, Dra. Elizabeth Gonzáleza ${ }^{a}$ Dr. Rodolfo P. Moreno ${ }^{a}$, Dra. Cecilia Seligra ${ }^{a}$, \\ Dr. Javier Fain ${ }^{a}$, Dr. Alberto Hernández ${ }^{a}$, Dra. Ivana Boailchuck ${ }^{b}$ y Dr. Gustavo Cardigni ${ }^{a}$
}

\begin{abstract}
RESUMEN
La ingestión involuntaria o la aspiración de un dispositivo o pieza de arco de los brackets y / u ortodoncias pueden constituir una emergencia médica con complicaciones potencialmente peligrosas. El objetivo de esta comunicación es describir una complicación poco frecuente del desplazamiento e ingesta posterior del arco de brackets.

Paciente de 13 años de edad, que sufrió la ruptura de la ortodoncia durante la alimentación, con la posterior ingesta de una parte. Padeció disfagia, que no mejoró a pesar de la ingesta de alimentos blandos. Consultó en el Servicio de Emergencias y presentó disfagia, sialorrea, dolor en la faringe y dificultad respiratoria leve. Se realizó la extracción del elemento de ortodoncia por vía endoscópica; se observó el alambre incrustado a nivel del polo inferior de la amígdala derecha y se extrajo. Se realizó, además, esofagoscopía, y no se observaron restos de este en el esófago. La paciente recibió el alta sin secuelas.
\end{abstract}

Palabras clave: ortodoncia, alambres para ortodoncia, endoscopía.

\section{ABSTRACT}

Involuntary ingestion or aspiration of a brace device and/ or orthodontic arch part can constitute a medical emergency with potentially dangerous complications. The objective of this communication is to describe a rare complication of posterior displacement and ingestion of the brace arch.

A 13-year-old girl, suffers from a break in orthodontia during feeding, with the subsequentingestion of part of it. She presented dysphagia after the fact; it did not improve despite the intake of soft foods. She consulted in the Emergency Department for dysphagia, sialorrhea, pain in the pharynx and mild respiratory distress. Extraction of the orthodontic element was performed endoscopically. The embedded wire was observed at the level of the lower pole of the right amygdala and it was removed. Esophagoscopy was also performed, not observing remains of it in the esophagus. The patient was discharged without sequelae. Key words: orthodontics, orthodontic wires, endoscopy.

a. Servicio de Pediatría.

b. Endoscopía Respiratoria Pediátrica.

Sanatorio de la Trinidad Palermo, Ciudad Autónoma de Buenos Aires, Argentina.

Correspondencia:

Dr. Rodolfo P. Moreno: morenopablorod@hotmail.com

Financiamiento: Ninguno.

Conflicto de intereses: Ninguno que declarar.

Recibido: 3-7-2020

Aceptado: 3-8-2020 http: / / dx.doi.org/10.5546/aap.2020.e558

Cómo citar: Labadens MS, González E, Moreno RP. Seligra C, et al. Lesión en la hipofaringe por un desplazamiento del arco de brackets. Reporte de caso. Arch Argent Pediatr 2020;118(6):e558-e561.

\section{INTRODUCCIÓN}

Los brackets son elementos metálicos que se cementan de forma fija en la superficie lingual o vestibular de los dientes y realizan su función gracias a un arco. Así, se generan fuerzas transmitidas a los dientes para reposicionarlos y corregir maloclusiones existentes.

Los episodios de ingestión o aspiración de un cuerpo extraño son complicaciones potenciales en todas las ramas de la odontología. ${ }^{1}$ La ingestión o inhalación accidental de cuerpos extraños ha sido ampliamente documentada, incluidos los incidentes que ocurren durante el tratamiento dental. La mayoría de los objetos ingeridos pasan a través del tracto gastrointestinal de forma espontánea, pero, aproximadamente, del $10 \%$ al $20 \%$ deben extraerse por vía endoscópica, y el $1 \%$ requiere cirugía. ${ }^{2}$

Los arcos ortodónticos o los aparatos fracturados que se tragan accidentalmente pueden alojarse en las vías respiratorias o en el tracto gastrointestinal. La ingestión involuntaria o la aspiración de un dispositivo o pieza de arco pueden constituir una emergencia médica con complicaciones potencialmente peligrosas, incluida la obstrucción de la vía aérea por un cuerpo extraño (OVACE). ${ }^{3}$ Se presenta una complicación poco frecuente del desplazamiento e ingesta posterior del arco de brackets.

\section{CASO CLÍNICO}

Paciente de 13 años de edad, que sufrió la ruptura de la ortodoncia durante la alimentación, con la posterior ingesta de una parte. Padeció disfagia, que no mejoró a pesar de la ingesta de alimentos blandos. Consultó en el Servicio de 
Emergencias y presentó disfagia, sialorrea, dolor en la faringe y dificultad respiratoria leve.

Se encontraba en buen estado general, molesta. Hemodinámicamente compensada. Tenía taquipnea leve, con buena entrada de aire bilateral. No se palpaba crepitación en la región del cuello. En la inspección de las fauces, no se observaban fragmentos de ortodoncia. Se internó para la evaluación y el estudio.

Se realizaron estudios complementarios: radiografía de cuello, proyecciones de frente y de perfil. En ambas, se observó, a nivel de la hipofaringe anterior, una pieza metálica alargada y fina, compatible con el elemento metálico de ortodoncia, que asomaba en la vía aérea. Las radiografías fueron tomadas 120 minutos después de que se produjo el incidente, e impresionaba que se habría producido la impactación (Figura 1).

Se interconsultó con Endoscopía Respiratoria y, con el diagnóstico de cuerpo extraño en la hipofaringe, se decidió realizar, como diagnóstico y eventual tratamiento, la evaluación endoscópica bajo anestesia en quirófano. La cavidad oral y la orofaringe se examinaron visualmente para descartar la posibilidad de que el arco quedara atrapado en dicha región. Se observó el alambre incrustado a nivel del polo inferior de la amígdala derecha, y se extrajo por vía endoscópica. Se realizó, además, esofagoscopía, y no se observaron restos de este en el esófago.
Con el laringoscopio, se constató que la laringe y la tráquea estaban libres.

La evolución clínica general fue muy buena, con buena tolerancia a la vía oral. Recibió profilaxis con antibióticos y analgesia. No hubo complicaciones infecciosas. Egresó al segundo día de internación, en buen estado general, sin signos de dificultad respiratoria o de toxoinfección, en seguimiento ambulatorio con su pediatra de cabecera y Endoscopía Respiratoria.

\section{DISCUSIÓN}

La ingestión o aspiración accidental de un objeto extraño es un riesgo siempre presente durante todos los procedimientos dentales, incluido el tratamiento de ortodoncia. El riesgo de inhalación o ingestión de objetos de ortodoncia es pequeño, y las consecuencias, rara vez, son graves. ${ }^{4} \mathrm{La}$ incidencia reportada de objetos ingeridos de origen dental varía considerablemente en la literatura, entre el 3,6\% y el $27,7 \%$ de todos los cuerpos extraños. ${ }^{5}$

La mayoría de los componentes de ortodoncia son pequeños, pero pueden ser difíciles de manejar. En ocasiones, los aparatos de ortodoncia o pequeños componentes de ortodoncia de estos se tragan accidentalmente, lo que genera problemas en las vías respiratorias o en el tracto gastrointestinal. ${ }^{6}$ Cerca del $90 \%$ de los objetos extraños ingeridos llega al tracto gastrointestinal.

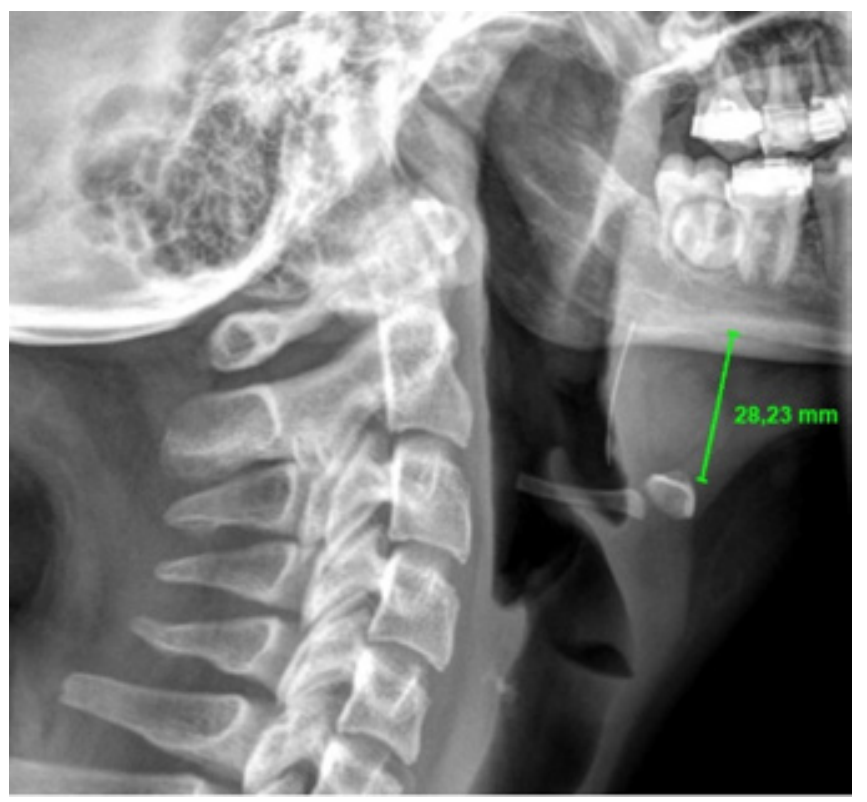


Hay alrededor de un $10 \%$ que requieren extirpación endoscópica, mientras que aun el $1 \%$ requerirá alguna cirugía.?

El tamaño, la forma y la presencia de bordes afilados del objeto ingerido influirán en los factores de riesgo, el manejo y el resultado. ${ }^{5}$ Los brackets pueden despegarse de la superficie dental y caerse durante la ingesta de alimentos duros. Con los movimientos dentales, los arcos se pueden desplazar y salirse más en un lado que en otro y pueden desprenderse. La fractura de los arcos permite que puedan aspirarse o tragarse. ${ }^{3}$ Esta situación suele darse en las primeras etapas del tratamiento de ortodoncia, cuando los dientes empiezan a moverse y el alambre se endereza. Sin embargo, también puede ocurrir en cualquier otro momento. Cuando el arco se desliza lateralmente, puede causar dolores y molestias en la parte posterior de la boca y la mejilla, que se tratan colocando cera de ortodoncia en el alambre.

La ingesta del arco es una complicación poco reportada y, menos aún, la impactación en la faringe. ${ }^{8}$ Aunque la ocurrencia es infrecuente, la morbilidad potencial asociada es muy alta. ${ }^{9} \mathrm{La}$ importancia del reconocimiento temprano de los signos de la obstrucción de las vías aéreas y el manejo rápido deben enfatizarse, y el paciente debe ser referido a un Servicio de Emergencias.

Los estudios de imagen son el enfoque inicial y son muy útiles para determinar la ubicación de cuerpos extraños ingeridos, pero tienen como limitaciones que los objetos pueden estar hechos de material radiolúcido y no ser visibles en la radiografía. Además, es posible que no se identifiquen pequeños objetos metálicos, y la ubicación del objeto puede ser inexacta. ${ }^{10}$ Aparte, no son de utilidad ante la presencia de síntomas de OVACE. En el caso presentado, la resolución rápida por vía endoscópica fue fundamental para evitar la migración del arco hacia la vía aérea y disminuir la posibilidad de obstrucción de esta. ${ }^{11}$

La literatura odontológica que aborda la aspiración o ingestión de instrumentos dentales, materiales o prótesis está repleta de informes de casos y descripciones de eventos adversos, ${ }^{1-7,9}$ pero no sucede lo mismo con la literatura pediátrica, $y$, por ello, es menos frecuente que los pediatras incluyan en su práctica diaria recomendaciones o medidas preventivas o de alarma cuando sus pacientes comienzan los tratamientos de ortodoncia. Con medidas preventivas y cuidados, la mayoría de estas complicaciones pueden limitarse o evitarse completamente ${ }^{8-10,12}$ (Tabla 1).

Finalizando, las lesiones relacionadas con la ruptura de los brackets son poco frecuentes, pero pueden provocar lesiones traumáticas graves. La morbilidad potencial asociada con cualquier incidente es demasiado alta para ignorarla. Se considera que es necesario realizar mayor difusión de las medidas preventivas a fin de evitar la presentación de nuevos casos.

\section{REFERENCIAS}

1. Milton TM, Hearing SD, Ireland AJ. Ingested foreign bodies associated with orthodontic treatment: report of three cases and review of ingestion/aspiration incident management. Br Dent J. 2001; 190(11):592-6.

2. Puryer J, McNamara C, Sandy J, Ireland T. An Ingested Orthodontic Wire Fragment: A Case Report. Dent J (Basel). 2016; 4(3):24.

3. Kumar Umesan U, Wizziyiane Ahmad W, Balakrishnan P. Laryngeal impaction of an archwire segment after accidental ingestion during orthodontic Adjustment. Am J Orthod Dentofacial Orthop. 2012; 142(2):264-8.

TABLA 1. Recomendaciones para pacientes con ortodoncia

- Evitar comer alimentos pegajosos, como chicles, caramelos, dulces, etc.

- Evitar comer alimentos duros, como caramelos, confites, chocolates, turrones, manzanas, etc. Estos pueden fracturar o despegar los brackets, alambres y aparatos.

- Mantener una excelente higiene bucal: tres cepilladas diarias después de las comidas. Además, se recomienda usar seda dental después de las comidas. El tiempo normal de cepillado de una persona que no lleva ortodoncia es de dos minutos; una persona que usa brackets deberá hacerlo durante tres-cinco minutos.

- Para los pacientes con brackets, hay cepillos especiales que les facilitan la higiene oral. Estos son diseñados para una limpieza eficiente y vienen integrados en los denominados kits de ortodoncia.

- Durante los primeros días, podría ocurrir alguna sensación de dolor. En este caso, preguntar al pediatra u ortodoncista cuál es el analgésico indicado. No automedicarse.

- Cuando un bracket o alambre ocasiona molestias en los labios o tejidos blandos, deberán colocarles cera o parafina.

- Si algún fragmento se cae o se despega, no autocolocarlo. Se recomienda solicitar un turno para su reparación.

- Asistir periódicamente a los controles odontológicos y pediátricos programados. 
4. Varho R, Oksala H, Tolvanen M, Svedström-Oristo AL. Inhalation or ingestion of orthodontic objects in Finland. Acta Odontol Scand. 2015; 73(6):408-13.

5. Kumar Umesan U, Lay Chua K, Balakrishnan P. Prevention and management of accidental foreign body ingestión and aspiration in orthodontic practice. Ther Clin Risk Manag. 2012; 8:245-52.

6. Handa A, Handa JK. Accidental ingestion of a foreign body of orthodontic origin-a review of risks, complications and clinical recommendations. Int J Orthod Milwaukee. 2016; 27(1):41-4.

7. Abusamaan M, Giannobile WV, Jhawar P, Gunaratnam NT. Swallowed and aspirated dental prostheses and instruments in clinical dental practice: a report of five cases and a proposed management algorithm. J Am Dent Assoc. 2014; 145(5):459-63.
8. Dibiase AT, Samuels RH, Ozdiler E, Akcam MO, Turkkahraman H. Hazards of orthodontics appliances and the oropharynx. J Orthod. 2000; 27(4):295-302.

9. HouR, ZhouH,HuK, Ding Y, etal. Thoroughdocumentation of the accidental aspiration and ingestion of foreign objects during dental procedure is necessary: review and analysis of 617 cases. Head Face Med. 2016; 12(1):23.

10. Cotrim J, Corujeira S, Jardim J, Cardoso H, et al. Accidental Ingestion of Dentistry Material - Report of Cases and Challenges from the Pediatrician Point of View. GE Port J Gastroenterol. 2014; 22(1):28-31.

11. Tripathi T, Rai P, Singh H. Foreign body ingestion of orthodontic origin. Am J Orthod Dentofacial Orthop. 2011; 139(2):279-83.

12. Gyocsi A, Kolarovszki B, Frank D. Adverse effects of orthodontic treatments. Fogorv Sz. 2016; 109(4):111-8. 